\title{
Multisegmental Herpes Zoster in a Healthy Girl
}

\section{Hussein Hassab EINaby ${ }^{1}$, Mohamed Ismail Kamel ${ }^{1}$ and Mohamed L Elsaie ${ }^{2,3 *}$}

${ }^{1}$ Department of Dermatology, Venereology and Andrology, Al Zhar University, Cairo, Egypt

${ }^{2}$ Department of Dermatology and Cutaneous Surgery, University of Miami, Florida

${ }^{3}$ Department of Dermatology and Venereology, National Research Center, Cairo, Egypt

\section{Abstract}

Children infrequently are afflicted with herpes zoster $(\mathrm{HZ})$. Activation of latent varicella-zoster virus (VZV) in a partially immune host results in $\mathrm{HZ}$. Herpes zoster in children can be benign or with varied severity, especially in cases associated with malignancy. Because of its rarity, we report widespread multisegmental $\mathrm{HZ}$ primarily presenting on the right side of the body and right flank in a 11 years old immunocompetent girl. The Authors have previously described a similar observation in a 6 years old female immunocompetent girl

Children infrequently are afflicted with herpes zoster $(\mathrm{HZ})$. Activation of latent varicella-zoster virus (VZV) in a partially immune host results in $\mathrm{HZ}$. Herpes zoster in children can be benign or with varied severity, especially in cases associated with malignancy. Defects in normal immunity and cellular immunity have been postulated as important factors in the pathogenesis of $\mathrm{HZ}$. Disseminated $\mathrm{HZ}$ is typical $\mathrm{HZ}$ associated with widespread cutaneous lesions simulating chicken pox and it may be associated with immunosuppression [1]

\section{Case Report}

A 6-year-old girl presented with a dermatomal blistering rash of 1 week's duration affecting the right side of her abdomen and back and right upper limb extending to the knee (Figures 1 and 2). The blisters were preceded by a 4 days history of low-grade fever, anorexia, malaise, and pain at the site where the blisters erupted. The patient did not have

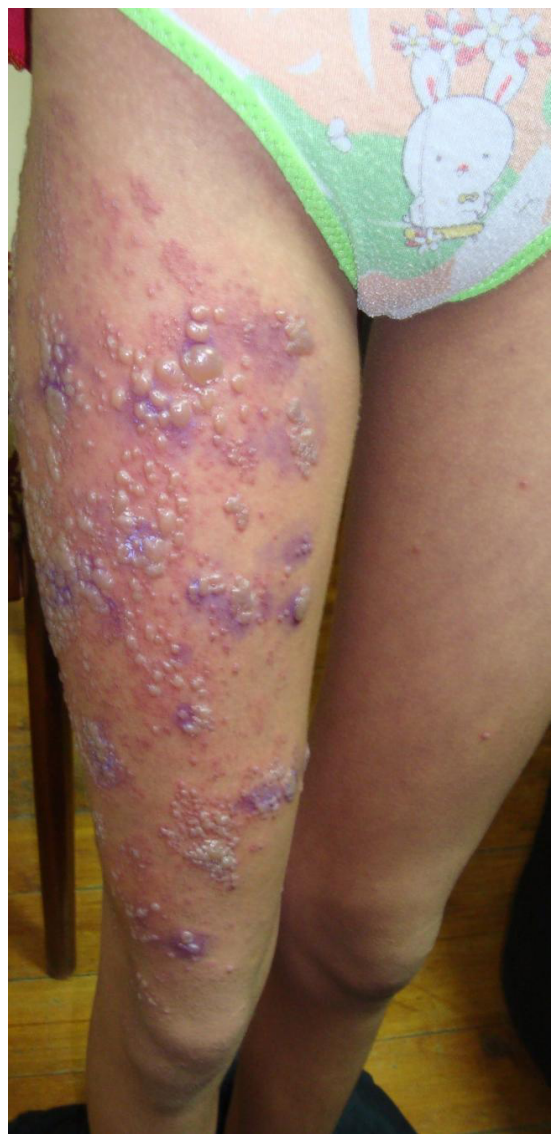

Figure 1: Disseminated vesicles on the right side of the body.

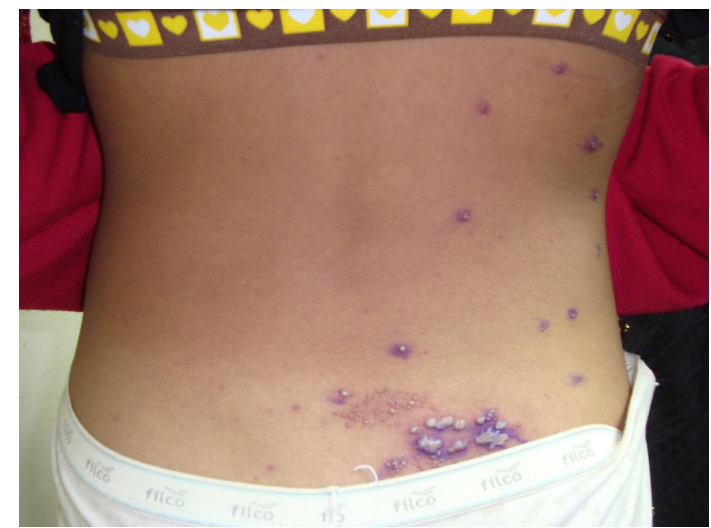

Figure 2: Vesicular eruption on the trunk.

a history of exposure to varicella (chickenpox) and had not received the varicella vaccine. The patient's mother had chickenpox as a child but could not recall the exact age of affection; however, she was certain that she had chickenpox before the patient was born. There was no evidence of the patient's predilection for other infections.

Physical examination showed confluent vesicles and bullae along the course of the right $\mathrm{T} 11$, and T12 nerves and extending on the right limb to the knees. There also were a few scattered vesicles with central dimples on the back.

Tzanck smear was performed by removing the crust from a vesicle and scraping the underlying moist skin with a No. 15 surgical blade. The cells were smeared from the vesicle base onto a slide, fixed for 1

*Corresponding author: Mohamed L Elsaie, Department of Dermatology and Cutaneous Surgery, University of Miami, Florida, USA, E-mail: egydoc77@@yahoo.com

Received April 04, 2013; Accepted April 23, 2013; Published April 26, 2013

Citation: EINaby HH, Kamel MI, Elsaie ML (2013) Multisegmental Herpes Zoster in a Healthy Girl. J Vaccines Vaccin 4: 180. doi:10.4172/2157-7560.1000180

Copyright: @ $2013 \mathrm{EINaby} \mathrm{HH}$, et al. This is an open-access article distributed under the terms of the Creative Commons Attribution License, which permits unrestricted use, distribution, and reproduction in any medium, provided the original author and source are credited. 
minute with absolute alcohol, and stained with Wright stain. The test showed multinucleated giant cells, while her complete blood cell counts showed mild leukocytosis with a relatively normal differential count. VZ count per vesicular fluid was 340,000 cells (N: up to 100,000 ). A complete metabolic profile of liver function test, kidney function tests, blood sugar was within normal limits. An enzyme-linked immunosorbent assay for human immunodeficiency virus screening was nonreactive and was repeated twice. A chest radiograph was normal and Immunoelectrophoresis was done and showed no evidence of any gammopathies.

The diagnosis of disseminated shingles in an immunocompetent 11 years old girl was made. The patient was prescribed acyclovir 800 mg 5 times daily for 7 days as an outpatient. The patient tolerated the medication well and her $\mathrm{HZ}$ resolved.

\section{Comment}

Patients present with unilateral pain followed by a unilateral vesicular rash that generally involves the skin of a single dermatome. The eruption begins as grouped vesicles on an erythematous base. These clusters of erythematous, round-to-oval lesions $(2-4 \mathrm{~mm}$ vesicles) measure several centimeters in diameter and are oriented along the track of dermatomal innervation. Over the ensuing days, the vesicles become pustules, and, finally, with rupture, grouped, crusted erosions are left. Lesions erupt over 7 days and develop a crust by 14-21 days. Thoracic dermatomes are the most common site. Involvement of multiple, contiguous dermatomes are not common.

Varicella-zoster virus infection causes 2 clinically distinct forms of disease: varicella (chickenpox) and HZ (shingles). Primary VZV infection results in the diffuse vesicular rash of varicella (or chickenpox). Clinical resolution is followed by the establishment of latent infection within the sensory dorsal root ganglia. Reactivation of this neurotropic virus leads to the secondary form known as HZ, or shingles, which is a painful unilateral vesicular eruption in a restricted dermatomal distribution.

The incidence of HZ has been well-studied. In the United States, HZ occurs in nearly 1 million people annually [2]. The cumulative lifetime incidence is approximately $10 \%$ to $20 \%$ of the population [3]. Overall, the Centers for Disease Control and Prevention estimates that $32 \%$ of individuals in the United States will experience zoster during their lifetime [4].

Age is the most important risk factor for the development of zoster. One study demonstrated that zoster incidence increased 10-fold with age when comparing children younger than 10 years to individuals aged 80 to 89 years; a dramatic increase in disease rates begins to occur after 50 years of age [5].

During an episode of primary varicella infection (chickenpox), VZV is highly contagious and spread both by respiratory droplets (sneezing and coughing) and direct contact. Infection with VZV occurs when the virus comes into contact with the mucosa of the upper respiratory tract or the conjunctiva of the eye. The virus travels in the bloodstream via mononuclear cells to the skin, resulting in the generalized rash of chickenpox. The virus also infects human cells in the dorsal root ganglia of the spinal column and cranial nerve ganglia where it becomes latent. Essentially protected from the human immune system, VZV typically remains dormant in the ganglia for decades. Herpes zoster occurs when the virus subsequently reactivates. Initially causing pain and soon afterwards a vesicular rash in the distribution of 1 or 2 contiguous dermatomes, it is less contagious than varicella. Zoster generally spreads only by direct contact with open draining lesions and not via airborne droplets [6].

Our patient is unique because she presented with the secondary infection of $\mathrm{HZ}$ without prior exposure to $\mathrm{VZV}$ and thus no primary infection. Elsaie et al. recently described a similar case in a 6 years old otherwise healthy child with no precious exposure to VZV. Primary infection of the individual with VZV results in varicella whereas reactivation of the latent virus lying dormant in the sensory ganglia results in HZ. In our case, the patient had no history of chickenpox, so disseminated $\mathrm{HZ}$ is difficult to explain. However, the primary infection may have been so mild it went unnoticed.

\section{Conclusion}

We report a unique case of widespread unilateral multisegmental $\mathrm{HZ}$ in a healthy 11 years-old girl who recovered uneventfully.

\section{References}

1. Mittal RR, Gill SS, Kaur K (1994) Unilateral multisegmental herpes zoster in a normal child. Indian J Dermatol Venereol Leprol 60: 362-363.

2. Yawn BP, Saddier P, Wollan PC (2007) A population-based study of the incidence and complication rates of herpes zoster before zoster vaccine introduction. Mayo Clin Proc 82: 1341-1349.

3. Straus SE, Ostrove JM, Inchauspe G, Felser JM, Freifeld A, et al. (1988) NIH conference. Varicella-zoster virus infections. Biology, natural history, treatment and prevention. Ann Intern Med 108: 221-237.

4. Harpaz R, Ortega-Sanchez IR, Seward JF, Advisory Committee on Immunization Practices (ACIP) Centers for Disease Control and Prevention (CDC) (2008) Prevention of herpes zoster: recommendations of the advisory committee on immunization practices (ACIP). MMWR Recomm Rep 57: 1-30.

5. Ragozzino MW, Melton LJ 3rd, Kurland LT, Chu CP, Perry HO (1982) Populationbased study of herpes zoster and its sequelae. Medicine (Baltimore) 61: 310 316.

6. Oxman MN, Alani R Varicella (1993) Dermatology in General Medicine. (4thedn). New York, NY: McGraw-Hill, 2543. 